\title{
Meta-analysis Derivation Concedes Clinical Significance in Democratisation of Healthcare
}

\author{
Mohamad Bashir ${ }^{1}$, Mohammed Idhrees ${ }^{2}$, Mostafa Mousavizadeh ${ }^{3}$, Yousef Rezaei ${ }^{4}$, and \\ Saeid Hosseini ${ }^{3}$ \\ ${ }^{1}$ Royal Blackburn Teaching Hospital \\ ${ }^{2}$ SRM Institutes for Medical Science Vadapalani \\ ${ }^{3}$ Rajaie Cardiovascular Medical and Research Center \\ ${ }^{4}$ Affiliation not available
}

July 14, 2021

\begin{abstract}
This is a response to letter, and does not contain the structured abstract

Meta-analysis Derivation Concedes Clinical Significance in Democratisation of Healthcare

Mohamad Bashir ${ }^{1}$, MD, Ph.D; Idhrees Mohammed ${ }^{2}$, MD; Mostafa Mousavizadeh ${ }^{3}$, MD; Yousef Rezaei ${ }^{3}$, MD; Saeid Hosseini ${ }^{3}$, MD

${ }^{1}$ Department of Vascular and Endovascular Surgery, Royal Blackburn Teaching Hospital, Blackburn, UK

${ }^{2}$ Institute of Cardiac and Aortic Disorders (ICAD), SRM Institutes for Medical Science (SIMS Hospital), Chennai, Tamil Nadu, India

${ }^{3}$ Heart Valve Disease Research Center, Rajaie Cardiovascular Medical and Research Center, Iran University of Medical Sciences, Tehran, Iran
\end{abstract}

Corresponding Author:

Mohamad Bashir, MD, PhD, MRCS

Department of Vascular and Endovascular Surgery

Royal Blackburn Teaching Hospital

Haslingden Rd, Blackburn BB23HH, UK

Email: drmbashir@mail.com

Keywords: Meta-analysis, publication bias, Meta-regression, aorta, frozen elephant trunk

To the Editor: We appreciate Wu and colleagues'1 letter submitted and entailed few critical points regarding the published meta-analysis data and the credibility of conclusions perceived. Thus, and in an attempt to demystify the confusion, we summarized few pointers that $\mathrm{Wu}$ et al. need to consider in interpretation of meta-analysis:

1. It's not a surprisingly practice that we pursue a rather scientific and stringent execution of thoughts and quality metric articles' assessment for clarity, quality, quantity, and critical appraisal components. Our 
extensive experience in publishing systematic reviews leans stringent data requirements to substantiate the objectives that had to be mandated. In contrary to what $\mathrm{Wu}$ et al ${ }^{1}$ perceived, publication bias is not a practice we follow to achieve desired outcomes. As such cognitive selective steps are adhered to and following quality metrics from published articles observed. The frequency of observational data declaring statistical significance in dire surgical experience as in Type A dissection is a reflection of current trends and practices. As such, it would be seemingly positive that publication bias is an unequivocal mannerism in constructing a rather niche meta-analysis. We have undoubtedly maintained a stream of thought to exclude any overlapped/duplicated population from the same institutions per se, alluding to extractable data for patients with isolated acute type A aortic dissection undergoing total arch replacement with frozen elephant trunk (FET). Concerns highlighted on this entity can easily be slotted into the following methodological nature of all meta-analyses. In concluding this section, as with all meta-analysis we published, we've implemented a dual-reviewer process including abstract screening upon selection of relevant studies as alluded to and aforementioned. ${ }^{2}$

2. We follow a rather constructive approach of heteroscedasticity to overcome culpable heterogeneity in all meta-analysis we conducted. The idea to give small weighted regression to observation associated with higher variances to minimize potential residual is a substantial practice we follow. As such postoperative stroke and SCI were found to be $\mathrm{I}^{2}=63.93 \%$ and $\mathrm{I}^{2}=19.56 \%$, respectively; however, we did not find any significant effect of subgroup analyses on the heterogeneity of pooled estimation of postoperative stroke. Our objectives and primary focus were on neurological outcomes, and as such, we performed a meta-regression analysis for those observed outcomes with variable heterogeneity. Additionally, there are many confounding factors that we were not able to extract from these observational studies to explore potential factors affecting the final meta-analysis. This is the limitation of the majority of a meta-analysis conducted. The lack of stringent criteria for randomization and the overwhelming unethical platform to conduct such studies which surely $\mathrm{Wu}$ et al ${ }^{1}$ would agree yield a rather homoscedasticity allowing a sequence of random variables to have a finite variance with time linear function. Compounding this notion is the lack of randomized clinical trials for FET procedure and the paucity of standard presentation of study results and baseline characteristics precluded us from a comprehensive exploration of confounding factors.

3. It's our practice that standardization and protocolization in meta-analysis are pursued, thus, we tried to extract data from the main text of the articles, however, we stumbled across different challenges that culpably allowed us to use a common denominator i.e. reading full-text, we used method section and the affiliation of the ethics committee for assigning the location of study institution, neither database affiliation and nor corresponding affiliations were a streamed thought amongst all contributors of this analysis. Moreover, Sun and colleagues, ${ }^{3}$ in their method section of their study had assigned "patients underwent surgical treatment at Fuwai Hospital, Beijing, China". Given this notion, we considered it as an affiliated study with Fuwai Hospital. It might be recommended that the adherence to the standards of the ethics committee and outcome reporting studies with a defined affiliation of the population would be imperative aiding future researchers and misleading endeavors. The large number of patients undergoing the FET procedure in Anzhen hospital has resulted in the publication of many articles with overlapped or even duplicated populations per se.

4. We have performed a univariate meta-regression to find a linear relationship between neurological outcomes and the utmost important variable associated with these events. Due to lack of data in the majority of studies and small sample size of included studies into the regression model, it is justifiable that implementation of a multivariate meta-regression analysis would be realistically unjustified and would render the desired characteristic and outcomes to be over-inflated disturbing our attempt to generate meaningful analysis in this evidence synthesis and precision healthcare era.

\section{References}

1. Wu J, Sun T, Fan R. Methodological quality of a meta-analysis precedes its clinical significance. J Card Surg . 2021

2. Gartlehner G, Affengruber L, Titscher V, Noel-Storr A, Dooley G, Ballarini N, König F. Single-reviewer 
abstract screening missed 13 percent of relevant studies: A crowd-based, randomized controlled trial. J Clin Epidemiol . 2020;121:20-28

3. Sun L, Qi R, Zhu J, Liu Y, Zheng J. Total arch replacement combined with stented elephant trunk implantation: A new "standard" therapy for type a dissection involving repair of the aortic arch? Circulation . 2011;123:971-978 